\title{
Exercise in obese pregnant women: The role of social factors, lifestyle and pregnancy symptoms
}

\author{
Katie F Foxcroft ${ }^{1{ }^{* *}}$, Ingrid J Rowlands ${ }^{2+}$, Nuala M Byrne ${ }^{3}, \mathrm{H}_{\text {David Mclntyre }}^{4}$, Leonie K Callaway ${ }^{5}$, \\ the BAMBINO group
}

\begin{abstract}
Background: Physical activity may reduce the risk of adverse maternal outcomes, yet there are very few studies that have examined the correlates of exercise amongst obese women during pregnancy. We examined which relevant sociodemographic, obstetric, and health behaviour variables and pregnancy symptoms were associated with exercise in a small sample of obese pregnant women.
\end{abstract}

Methods: This was a secondary analysis using data from an exercise intervention for the prevention of gestational diabetes in obese pregnant women. Using the Pregnancy Physical Activity Questionnaire (PPAQ), 50 obese pregnant women were classified as "Exercisers" if they achieved $\geq 900 \mathrm{kcal} / \mathrm{wk}$ of exercise and "Non-Exercisers" if they did not meet this criterion. Analyses examined which relevant variables were associated with exercise status at 12, 20, 28 and 36 weeks gestation.

Results: Obese pregnant women with a history of miscarriage; who had children living at home; who had a lower pre-pregnancy weight; reported no nausea and vomiting; and who had no lower back pain, were those women who were most likely to have exercised in early pregnancy. Exercise in late pregnancy was most common among tertiary educated women.

Conclusions: Offering greater support to women from disadvantaged backgrounds and closely monitoring women who report persistent nausea and vomiting or lower back pain in early pregnancy may be important. The findings may be particularly useful for other interventions aimed at reducing or controlling weight gain in obese pregnant women.

\section{Background}

Physical activity during pregnancy is important for women's general health and may reduce the risk of adverse maternal, fetal and neonatal outcomes. Current recommendations advise pregnant women without medical or obstetric complications to aim for 30 minutes of physical activity on most days of the week [1]. Randomised controlled trials have shown that the uptake of regular exercise among sedentary pregnant women has significant benefits for women in pregnancy. Specifically, women who participated in three hours of weekly vigorous exercise in pregnancy reported greater satisfaction with their physical stamina, energy levels, appearance

\footnotetext{
* Correspondence: katie_foxcroft@health.qld.gov.au

+ Contributed equally

'Department of Internal Medicine and Aged Care, Royal Brisbane and

Women's Hospital, (Butterfield St), Brisbane, (4029), Australia

Full list of author information is available at the end of the article
}

and general health than sedentary pregnant women [2]. In another study of pregnant women who were overweight, participation in three hours of aerobic exercise per week was associated with higher fitness levels as demonstrated by increased oxygen uptake, than overweight women who remained sedentary [3]. Exercise also appears to have benefits for neonates, with the uptake of moderate-intensity exercise in pregnancy being associated with normal fetal growth [4]. However, some women may have difficulty meeting current recommendations, or participating in physical activity altogether during pregnancy because of health and psychosocial factors.

The correlates of physical activity among women during pregnancy have been examined in only a few studies. Sociodemographic variables such as education and income [5-8] have been positively associated with physical activity in pregnancy whereas a negative association

\section{Biomed Central}


has been found for age [7,9]. Women who have children at home and an unfavourable pregnancy history [9] are less likely to be physically active during pregnancy. Although there is limited evidence, psychosocial variables such as employment during pregnancy and lack of childcare [10] have also been identified as correlates of physical activity in pregnancy [11]. However, overall the evidence tends to be conflicting with several studies finding no relationship between these variables and women's physical activity levels during pregnancy.

Women's physical health and health behaviours before and during pregnancy may be important predictors of physical activity during pregnancy. Women who have a high pre-pregnancy body mass index (BMI) [11] or who smoke [6,7] are less likely to be physically active in pregnancy. On the other hand, pre-pregnancy physical activity has been associated with remaining physically active during pregnancy [10]. Remaining active during pregnancy may be beneficial for women's well-being, with one study showing that exercise during the first trimester of pregnancy was related to reduced reporting of nausea and vomiting in the $2^{\text {nd }}$ trimester of pregnancy [12].

Physical symptoms are common and normal in pregnancy, but they may deter or prevent some women from exercising during pregnancy. For example, almost $80 \%$ of pregnant women experience nausea and vomiting [12] in their first trimester. Although this generally resolves by the 12 th week of pregnancy, around $40 \%$ of women report nausea and vomiting into their second trimester and some women are affected for the entire pregnancy. Further, back pain during pregnancy affects anywhere between 24 and $90 \%$ of women and may interfere with women's ability to exercise $[13,14]$. While pregnancy symptoms may have a large impact on women's wellbeing, there is evidence to suggest that exercise may improve women's symptoms [8].The correlates of exercise during pregnancy among women who are obese have not been examined. Women who are obese are at the greatest risk for pregnancy complications [15] and weight retention in the longer term [16], and thus it seems important to examine whether there are factors that are common to obese pregnant women who do exercise. This study examines the correlates of exercise in a small sample of pregnant women who were identified as achieving, or not achieving adequate exercise-specific energy expenditure requirements throughout their pregnancy. We expected that the relevant sociodemographic, obstetric and health behaviour variables, and pregnancy symptoms, would be associated with exercise during pregnancy in this group of women. This information would be useful for informing lifestyle interventions that aim to reduce or control weight gain among obese pregnant women.

\section{Methods}

\section{Participants}

This is a secondary analysis of a study of 50 women who were recruited as part of a randomised controlled trial (RCT) examining the feasibility of an individualised exercise program for obese women during pregnancy. Full details of study design and participant recruitment have been reported previously [17]. Women receiving antenatal care and delivering at the Royal Brisbane and Women's Hospital (RBWH) in Queensland, Australia, were recruited from the hospital's maternity outpatient clinic between 12 and 14 weeks gestation. Ethics clearance was obtained for the study from the Royal Brisbane and Women's Hospital (RBWH) Human Research Ethics Committee. The RCT study is registered with the Australian Clinical Trials Registry (ACTRN012606000271505). Women were included in the study if they were: aged 18-45; had a BMI of $30 \mathrm{~kg}$. $\mathrm{m}^{-2}$ or greater; were willing to participate in an exercise program; and able to provide informed consent. Exclusion criteria included: non-English speaking; contraindication or inability to exercise; medical or obstetric contraindication to exercise including hemodynamically significant heart disease; restrictive lung disease; incompetent cervix (cerclage); multiple gestation; severe anaemia; chronic bronchitis; type 1 diabetes; orthopaedic limitations; poorly controlled seizure disorder; poorly controlled hyperthyroidism; heavy smoker.

Pre-intervention Stage. All eligible women were invited to attend a single early group education session at around 12 week's gestation. Women received written information on exercise [1], nutrition [18] and weight gain during pregnancy [19]. The women were subsequently invited to attend a face-to-face interview with a research midwife. Interviews collected information on demographics and physical and mental health and health behaviours.

\section{Intervention}

Women randomized to the intervention received a) an individualised exercise plan $b$ ) regular exercise advice and c) paper-based diaries for self-monitoring. A faceto-face interview at $\approx 12$ weeks with a physiotherapist, who had expertise in pregnancy management and exercise physiology, was conducted to develop women's individualized exercise plans; to assess readiness for change, and encourage goal setting. Women were reviewed every 4 weeks by physiotherapists, with phone calls between visits to assess their adherence to the program. Women who were not meeting exercise targets had additional face-to-face support, with identification of barriers and modification of the exercise plan.

Both the intervention and control groups were followed up at 12, 20, 28 and 36 weeks by a research midwife who 
recorded their weight, pregnancy symptoms and administered the PPAQ.

\section{Energy Expenditure}

We examined the correlates of energy expenditure, which are expressed as kilocalories per week (kcal) in this paper. Energy expenditure was derived from the Pregnancy Physical Activity Questionnaire (PPAQ). Data was collected at 12, 20, 28 and 36 weeks gestation.

\section{Pregnancy physical activity questionnaire (PPAQ; [20])}

This is a self-report instrument which measures the time spent participating in 32 activities including household/care giving, occupational, sports/exercise, transportation, and inactivity. The PPAQ is reliable and valid measure of exercise during pregnancy. Specifically, the intraclass correlation coefficient for the sports and exercise activity subscale was 0.83 , and scores for the sports and exercise subscale correlate moderately with actigraph data [20].

From the PPAQ, we extracted data for sports and exercise activities only. The types of sports and exercise activities assessed in the PPAQ include walking, jogging, prenatal exercise classes, swimming and dancing. To calculate weekly energy expenditure using the PPAQ, the duration of time spent in these exercise activities was multiplied by specific intensities (i.e. MET values) and scores are expressed as MET-hours per week.

In order take into account the women's weight, which can greatly affect energy expenditure, weekly kilocalories (kcals) expended by the women during exercise was calculated. Weekly kcals at each time point were derived by multiplying MET-hours per week by weight $(\mathrm{kg})$. Because the data was severely skewed, it was converted into categorical outcome variables at each time point. Because there are no recommended cut-offs, we chose the cut-point of $900 \mathrm{kcal} / \mathrm{wk}$ based the results of prior exercise intervention delivered to non-pregnant, obese individuals [21]. Thus, at each time point, women who achieved $<900 \mathrm{kcal} / \mathrm{wk}$ were classified as "Non-exercisers" and women who achieved $>900 \mathrm{kcal} /$ wk were classified as "Exercisers".

\section{Predictor Variables \\ Background information}

A semi-structured interview was used to collect information on maternal sociodemographic, obstetric, and health and health behaviour characteristics. The woman's height was measured at this interview and using self-reported pre-pregnancy weight, pre-pregnancy BMI was calculated.

\section{Pregnancy symptoms}

Women were asked to describe their pregnancy symptoms at 12, 20, 28 and 36 weeks. Based on women's qualitative descriptions of their symptoms at each time point, separate dichotomous variables were created for nausea/ vomiting and fatigue. For each symptom, women who reported having had the symptom were coded as "Yes", and women without this symptom were coded as "No".

The number of symptoms reported by women was also used to create variables showing the total number of symptoms reported at each time point. These variables were categorised as: $0-1 ; 2-3 ; 4$ or more.

\section{Low back pain}

The Roland-Morris Disability questionnaire (RDQ-24) is a self-report questionnaire assessing the level of physical disability resulting from low back pain. The questionnaire contains 24 statements describing symptoms of low back pain, and individuals are asked tick only those statements which apply to them on the day of completion. Total scores range from 0 to 24, with higher scores indicating greater disability. The RDQ-24 has been shown to be a reliable measure of low back pain that has been validated in a number of populations and countries. Internal consistency for the scale is good with Cronbach's alpha ranging from 0.84 to 0.93 [22].

\section{Statistical Analysis}

All analyses were conducted using STATA version 10.0 (StataCorp, Texas, USA). Univariate differences between the groups on the sociodemographic, obstetric, physical and mental health and health-related variables, and pregnancy symptoms, were examined using chi-square tests for independence and Fisher's exact tests for categorical variables, and unpaired t-tests for continuous variables. Women were included in the analyses at each time point if they had data on the PPAQ.

\section{Results}

\section{Participant Characteristics}

The mean age of the sample $(n=50)$ was $30 \pm 5$ yrs and slightly more than half of women were married and had at least one child (Table 1). Most women (74\%) reported being in either part-time or full-time employment at 12 weeks gestation and 30\% were tertiary educated. Almost one fifth of women had a history of miscarriage and $26 \%$ had had a previous caesarean section. The median prepregnancy weight was $90 \mathrm{~kg}$ and $36 \%$ of women had a BMI greater than $35 \mathrm{~kg} . \mathrm{m}^{-2}$, but smoking rates were relatively low (6\%). At 12 weeks gestation, nausea and fatigue affected $78 \%$ and $54 \%$ of women, respectively. Back pain affected $6 \%$ of women at 12 weeks and increased to $30.5 \%$ at 36 weeks gestation. Characteristics of the women according to their group allocation in the randomised control trial have been published elsewhere [17].

\section{Energy Expenditure}

Table 1 shows the proportion of women who were classified as Exercisers and Non-exercisers. At 12 weeks 
Table1 Sociodemographic, health, obstetric, and health behaviour predictors for women classified, Exercisers and Non-exercisers during pregnancy

\begin{tabular}{|c|c|c|c|c|c|c|c|c|}
\hline \multirow{3}{*}{ VARIABLE } & \multicolumn{2}{|c|}{12 weeks $^{4}$} & \multicolumn{2}{|c|}{20 weeks $^{4}$} & \multicolumn{2}{|c|}{28 weeks $^{4}$} & \multicolumn{2}{|c|}{36 weeks $^{4}$} \\
\hline & \multirow{2}{*}{$\begin{array}{l}\quad n=33 \\
\text { Non- } \\
\text { Exercisers }\end{array}$} & \multirow{2}{*}{$\begin{array}{c}n=17 \\
\text { Exercisers }\end{array}$} & \multirow{2}{*}{$\begin{array}{l}\quad n=18 \\
\text { Non- } \\
\text { Exercisers }\end{array}$} & \multirow{2}{*}{$\begin{array}{c}n=24 \\
\text { Exercisers }\end{array}$} & \multirow{2}{*}{$\begin{array}{l}\quad n=17 \\
\text { Non- } \\
\text { Exercisers }\end{array}$} & \multirow{2}{*}{$\begin{array}{c}n=24 \\
\text { Exercisers }\end{array}$} & \multirow{2}{*}{$\begin{array}{l}\quad n=21 \\
\text { Non- } \\
\text { Exercisers }\end{array}$} & \multirow{2}{*}{$\begin{array}{c}n=15 \\
\text { Exercisers }\end{array}$} \\
\hline & & & & & & & & \\
\hline $\begin{array}{l}\text { RCT study group } \\
\text { Control Intervention }\end{array}$ & $\begin{array}{l}18(55 \%) \\
15(45 \%)\end{array}$ & $\begin{array}{c}7(41 \%) \\
10(59 \%)\end{array}$ & $\begin{array}{l}10(63 \%) \\
6(38 \%)\end{array}$ & $\begin{array}{c}9(38 \%) \\
15(63 \%)\end{array}$ & $\begin{array}{l}11(65 \%) \\
6(35 \%)\end{array}$ & $\begin{array}{c}8(33 \%) \\
16(67 \%)\end{array}$ & $\begin{array}{l}11(55 \%) \\
9(45 \%)\end{array}$ & $\begin{array}{c}5(33 \%) 10 \\
(67 \%)\end{array}$ \\
\hline$p$ & \multicolumn{2}{|c|}{$0.37^{3}$} & \multicolumn{2}{|c|}{$0.12^{3}$} & \multicolumn{2}{|c|}{$0.047^{3}$} & \multicolumn{2}{|c|}{$0.20^{3}$} \\
\hline Age $M(S D)$ & $30.0(5.7)$ & $30.5(4.7)$ & $29.6(5.4)$ & $30.3(5.2)$ & $31.9(4.5)$ & $29.1(5.4)$ & $29.4(5.4)$ & $32.1(4.4)$ \\
\hline$p$ & \multicolumn{2}{|c|}{0.39} & \multicolumn{2}{|c|}{0.70} & \multicolumn{2}{|c|}{0.09} & \multicolumn{2}{|c|}{0.12} \\
\hline Tertiary & $10(30 \%)$ & $5(30 \%)$ & $4(22 \%)$ & $11(46 \%)$ & $5(29 \%)$ & $10(42 \%)$ & $4(19 \%)$ & $9(60 \%)$ \\
\hline$p$ & \multicolumn{2}{|c|}{$0.95^{1}$} & \multicolumn{2}{|c|}{$0.19^{3}$} & \multicolumn{2}{|c|}{$0.42^{1}$} & \multicolumn{2}{|c|}{$0.02^{3}$} \\
\hline \multicolumn{9}{|l|}{ Marital Status } \\
\hline Married & $24(73 \%)$ & $9(53 \%)$ & $10(56 \%)$ & 17 (71\%) & $9(53 \%)$ & 17 (71\%) & $13(62 \%)$ & $10(67 \%)$ \\
\hline$p$ & \multicolumn{2}{|c|}{$0.16^{1}$} & \multicolumn{2}{|c|}{$0.31^{1}$} & \multicolumn{2}{|c|}{$0.24^{1}$} & \multicolumn{2}{|c|}{$0.77^{1}$} \\
\hline Employed & $24(73 \%)$ & $13(76 \%)$ & $16(89 \%)$ & 17 (71\%) & $16(94 \%)$ & 17 (81\%) & $18(86 \%)$ & $11(73 \%)$ \\
\hline$p$ & \multicolumn{2}{|c|}{$0.77^{3}$} & \multicolumn{2}{|c|}{$0.29^{3}$} & & & & \\
\hline$\geq 1$ child at home & $18(55 \%)$ & $12(71 \%)$ & 7 (39\%) & 17 (81\%) & $8(47 \%)$ & $16(67 \%)$ & $14(67 \%)$ & $9(60 \%)$ \\
\hline$p$ & 0.2 & & & & & & & \\
\hline Previous miscarriages & $3(9 \%)$ & $6(35 \%)$ & $1(6 \%)$ & $6(25 \%)$ & $1(6 \%)$ & $6(25 \%)$ & $3(14 \%)$ & $4(27 \%)$ \\
\hline$p$ & 0.0 & & & & & & & \\
\hline Previous caesarean section & $8(24 \%)$ & $5(2(\%)$ & $3(17 \%)$ & $7(33 \%)$ & $3(18 \%)$ & $7(29 \%)$ & $3(14 \%)$ & $6(40 \%$ \\
\hline$p$ & 0.6 & & & & & & & \\
\hline Smoker & $3(9 \%)$ & $0(0 \%)$ & $2(11 \%)$ & $1(4 \%)$ & $2(12 \%)$ & $0(0 \%)$ & $2(10 \%)$ & $0(0 \%)$ \\
\hline$p$ & 0.5 & & & & & & & \\
\hline Pre-pregnancy weight & $93(75-176)$ & $85(68-95)$ & $92(75-176)$ & $\begin{array}{l}87.5(68- \\
105)\end{array}$ & $93(68-176)$ & $\begin{array}{c}87.5(78- \\
115)\end{array}$ & $93(68-175)$ & $\begin{array}{c}89(78.2- \\
176)\end{array}$ \\
\hline$p$ & 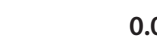 & & & & & & & \\
\hline $\begin{array}{l}\text { History of mental health } \\
\text { diagnoses }\end{array}$ & $11(33 \%)$ & $8(47 \%)$ & $7(39 \%)$ & $8(33 \%)$ & $6(35 \%)$ & $8(33 \%)$ & $9(43 \%)$ & $4(27 \%)$ \\
\hline$p$ & 0.3 & & & & & & & \\
\hline
\end{tabular}

Note. ${ }^{1}$ Chi-sqaure test. ${ }^{2}$ Mann-Whitney test. Data for pre-pregnancy weight is presented as: Median (range). ${ }^{3}$ Fisher's exact test. ${ }^{4}$ Numbers may not sum to total because some data missing

gestation, the proportion of Non-exercisers (66\%) was almost double that of Exercisers (34\%). Therefore, more than half of the women were not achieving greater than $900 \mathrm{kcal}$ of exercise per week. Although there was a marked decrease in the proportion of Non-exercisers at $20(40 \%)$ and 28 (41\%) weeks, the percentage of NonExercisers increased to $57 \%$ at 36 weeks. Data derived from the semi-structured interviews with the women conducted as part of the RCT showed that walking was the preferred method of exercise over the course of the trial, although a wide variety of activities were undertaken [23].

\section{Predictors of Exercise in Early Pregnancy}

Table 1 shows the selected sociodemographic, obstetric, health and health behaviour predictors of exercise at 12 weeks gestation for women who were classified Exercisers and Non-exercisers. Pre-pregnancy weight and a history of previous miscarriages were associated with exercise status. Exercisers were more likely to have a lower pre-pregnancy weight $(z=2.74, p=0.006)$ and a history of miscarriage than non-exercisers $(p=0.047$, Fisher's exact test). The number of children living at home was also a determinant of exercise status. At 20 weeks, Exercisers were more likely to have at least one child living at home than Non-exercisers $\chi^{2}(d f=1, N=$ 42) $=4.29$,

$$
p=0.038 \text {. }
$$

\section{Predictors of Exercise in Late Pregnancy}

Educational attainment was a significant predictor of exercise in late pregnancy (see Table 1). At 36 weeks, women who had completed tertiary education were three times more likely to be an Exerciser ( $p=0.02$, Fisher's exact test). At 28 weeks, women who were randomised to the intervention group in the RCT were more likely to be classified as an Exerciser $\chi^{2}(d f=1$, $N=41)=3.94, p=0.047$, and there was also a trend towards Exercisers being slightly younger than the NonExercisers ( $p=0.09$, Fisher's exact test). 


\section{Pregnancy Symptoms}

Self-reported pregnancy symptoms were associated with exercise status during pregnancy (Table 2). Specifically, women classified as Exercisers were less likely to report nausea or vomiting at 28 weeks gestation than Nonexercisers ( $p=0.01$, Fisher's exact test). Differences between the Exercisers and Non-exercisers were also found for low back pain. The distributions of low back pain for the Exercisers and Non-exercisers showed that Exercisers were more likely to report fewer symptoms of back pain than Non-exercisers $(z=1.99, p=0.047)$. There were no significant differences between the groups for fatigue and poor sleep at any point during pregnancy.

\section{Discussion}

The aim of the study was to examine the correlates of exercise among obese women in early and late pregnancy. Although relevant sociodemographic, health behaviour variables and pregnancy symptoms were associated with exercise status in this study, the only obstetric variable to show an association with exercise status was previous miscarriages. Women who had a history of miscarriage were more likely to be Exercisers very early in pregnancy. This is likely to reflect the fact that many pregnant women who have a history of pregnancy loss are anxious about future loss and may subsequently adopt healthier lifestyles in an attempt to prevent future miscarriages $[24,25]$.

Sociodemographic variables, including the number of children living at home and education were associated with exercise status in this study. We found that women with at least one child living at home were more likely to be classified as Exercisers, which is contrary to other evidence showing that women with children at home are less likely to be physically active $[6,9,10]$. It is possible that the women in our study with children were also not currently working, or had children who were older and in school. This may have allowed the women to have more time to exercise accounting for our findings. We found that education was a predictor of exercise status in late pregnancy. Consistent with previous findings $[6,8,9,26]$, women classified as Exercisers were more likely to have completed tertiary education than Non-exercisers. Our findings are not unexpected considering that the link between obesity and low socioeconomic status has been previously established [27]. Social disadvantage is associated with a range of poor health behaviours, and thus obese pregnant women who come from disadvantaged backgrounds may benefit from greater intervention. Pre-conception counselling may be particularly important for this group of women. However, low socio-economic status is generally associated with poor access to health services and this, in combination with the low rates of planned pregnancies [28], suggests that community-based interventions for these women may need to be considered as a feasible alternative. The fact that women assigned to the intervention group in the RCT were more likely to be classified as Exercisers in late pregnancy suggests the importance of providing support for obese pregnant women to facilitate long-term health behaviour change.

Pregnancy symptoms were also associated with exercise status during pregnancy. Women who reported lower back pain at 12 and 20 weeks and nausea or vomiting at 20 and 28 weeks were less likely to be classified as Exercisers. These findings are consistent with other evidence suggesting that exercise in early pregnancy is related to decreased reporting of nausea and vomiting in late pregnancy [12]. However, the direction

Table 2 Number of pregnancy symptoms: nausea, vomiting, fatigue, low back pain during pregnancy in PPAQ Exercisers versus PPAQ Non-exercisers

\begin{tabular}{|c|c|c|c|c|c|c|c|c|}
\hline \multirow[b]{2}{*}{ VARIABLE } & \multicolumn{2}{|c|}{12 weeks $^{4}$} & \multicolumn{2}{|c|}{20 weeks $^{4}$} & \multicolumn{2}{|c|}{28 weeks $^{4}$} & \multicolumn{2}{|c|}{36 weeks $^{4}$} \\
\hline & $n=33$ & $n=17$ & $n=18$ & $n=24$ & $n=17$ & $n=24$ & $n=21$ & $n=15$ \\
\hline No. of Symptoms & Non-exercisers & Exercisers & Non-Exercisers & Exercisers & Non-Exercisers & Exercisers & Non-Exercisers & Exercisers \\
\hline $0-1$ & $9(27 \%)$ & $4(24 \%)$ & $5(28 \%)$ & 14 (58\%) & $4(24 \%)$ & $10(42 \%)$ & $2(10 \%)$ & $3(20 \%)$ \\
\hline $2-3$ & $17(52 \%)$ & $11(65 \%)$ & $10(56 \%)$ & $8(33 \%)$ & $7(41 \%)$ & $12(50 \%)$ & $14(70 \%)$ & $9(60 \%)$ \\
\hline 4 or more & $7(21 \%)$ & $2(12 \%)$ & $3(17 \%)$ & $2(8 \%)$ & $6(35 \%)$ & $2(8 \%)$ & $4(20 \%)$ & $3(20 \%)$ \\
\hline$p$ & \multicolumn{2}{|c|}{$0.72^{3}$} & \multicolumn{2}{|c|}{$0.15^{3}$} & \multicolumn{2}{|c|}{$0.09^{3}$} & \multicolumn{2}{|c|}{$0.88^{3}$} \\
\hline Nausea \& Vomiting & $3(14 \%)$ & $6(37 \%)$ & $8(44 \%)$ & $3(13 \%)$ & $7(41 \%)$ & $1(4 \%)$ & $1(5 \%)$ & $2(13 \%)$ \\
\hline$p$ & \multicolumn{2}{|c|}{$0.136^{3}$} & \multicolumn{2}{|c|}{$0.033^{3}$} & \multicolumn{2}{|c|}{$0.005^{3}$} & \multicolumn{2}{|c|}{$0.565^{3}$} \\
\hline Fatigue & $17(52 \%)$ & $11(65 \%)$ & $3(17 \%)$ & $9(38 \%)$ & 7 (41\%) & $3(13 \%)$ & $3(15 \%)$ & $2(13 \%)$ \\
\hline \multirow[t]{2}{*}{$p$} & \multicolumn{2}{|c|}{$0.37^{1}$} & \multicolumn{2}{|c|}{$0.18^{3}$} & \multicolumn{2}{|c|}{$0.63^{3}$} & \multicolumn{2}{|c|}{$1.00^{3}$} \\
\hline & $n=32$ & $n=16$ & $n=14$ & $n=23$ & $n=15$ & $n=23$ & $n=21$ & $n=14$ \\
\hline Back Pain & $1(0-15)$ & $0(0-7)$ & $3(0-14)$ & $0(0-10)$ & $1(0-8)$ & $2(0-18)$ & $3(0-19)$ & $2(0-24)$ \\
\hline$p$ & \multicolumn{2}{|c|}{$0.006^{2}$} & \multicolumn{2}{|c|}{$0.03^{2}$} & \multicolumn{2}{|c|}{$0.30^{2}$} & \multicolumn{2}{|c|}{$0.25^{2}$} \\
\hline
\end{tabular}

Note. ${ }^{1}$ Chi-sqaure test. ${ }^{2}$ Mann-Whitney test. Data for back pain is presented as: Median (range). ${ }^{3}$ Fisher's exact test. ${ }^{4}$ Numbers may not sum to total because some data missing. 
of the relationship between these pregnancy symptoms and exercise in our study was not clear. The findings may suggest that exercise helps alleviate nausea and vomiting in pregnancy. Alternatively, women may have chosen to exercise because they experienced less nausea or vomiting during their pregnancy. In this study we found physical health differences between the Exercisers and Non-Exercisers. Specifically, pre-pregnancy weight in the Exercisers was lower at 12 weeks gestation than in the Non-exercisers. The differences between the groups for back pain found in early pregnancy also support this, and are consistent with evidence suggesting that pre-pregnancy physical activity is associated with a reduced risk of back pain during pregnancy [14]. Further work is required to determine if this is a causal relationship. In our study, it is unclear whether women who have less back pain are more likely to exercise, or whether women who do exercise benefit from a reduction in back pain.

A major issue with lifestyle interventions is the assessment of physical activity. Both subjective and objective measures of physical activity have well known limitations (27). The PPAQ was very useful measure in our study - easy to complete and tailored to measure physical activity among women during pregnancy. The selfreport nature of the questionnaire meant that we relied on the women to accurately recall their activity, and this may have led to an overestimation of exercise hours. Thus, our results may not generalise to other studies, particularly those relying on objective measures of exercise.

In the RCT, a number of women withdrew from the trial at different stages, limiting the data that was available for analysis over the course of the trial. A total of five women ( $n=3$, control; $n=2$, intervention) dropped out soon after their baseline visit. Three women withdrew from the trial when they discovered they had been randomised to the control group and were disappointed with this outcome. Two women who were randomised to the exercise arm also withdrew at 12 weeks when they were diagnosed with gestational diabetes based on their baseline blood tests. Among the remaining women, those that withdrew from the study ( $n=4$, control; $n=$ 5 , intervention) did so because of medical or obstetric complications (e.g. miscarriage, intrauterine fetal death, sacroiliac joint instability, gestational diabetes); five women delivered before 36 weeks and thus did not have data collected at 36 weeks. The retention rates in the RCT and the reasons for non-completion have been published elsewhere [18].

The small sample size in our study limited our ability to adjust for other variables, including age and pre-pregnancy BMI. The women who remained in the study may represent a highly motivated group, which may limit the extent to which these results generalise. The results of this small pilot study suggest that it may be important to adjust for sociodemographic variables (e.g. age, education), as well as pre-pregnancy BMI in future analyses examining the correlates of exercise in pregnancy.

\section{Conclusions}

This is the first study to identify the correlates of exercise during pregnancy in an obese population. We found that health-related variables tended to predict exercise in early pregnancy whereas sociodemographic variables were most likely to predict exercise in late pregnancy. Specifically, women who had a history of miscarriage; a lower pre-pregnancy BMI; who reported no nausea and vomiting; and who had no lower back pain, were those women who were most likely to have exercised in early pregnancy. Exercise in late pregnancy was most common among women who were better educated, and there was a trend for younger women to be classified as Exercisers during the third trimester of pregnancy. However, the direction of the relationship between exercise and pregnancy symptoms is unclear and requires further examination using larger samples prospectively designed to answer these questions.

The findings may be particularly useful for researchers who are designing interventions aimed at reducing or controlling weight gain in obese pregnant women. Interventions that offer greater support to women from disadvantaged backgrounds, and closer monitoring of women who report persistent nausea and vomiting or lower back pain during pregnancy may be beneficial. Providing this care and support may be an initial step towards increasing obese women's participation in exercise during pregnancy.

\section{Acknowledgements}

We would like to thank the entire BAMBINO group from the Royal Brisbane and Women's Hospital, Brisbane, Australia, for their contribution to the project - Susie Croaker, Dietitian- Nutritionist, Department of Nutrition and Dietetics - Xanthe Sansome, physiotherapist and research assistant - Briony O'Connor, physiotherapist and Ainsley Groves, exercise physiologist, School of Human Movement Studies, QUT.

†The complete BAMBINO Group: Leonie K.Callaway, Paul B.Colditz, Nuala M. Byrne, Barbara E. Lingwood, Ingrid J. Rowlands, Ainsley Groves, Xanthe Sansome, Briony R. O'Connor, Susan Croaker, Katie Foxcroft, and H.David Mclntyre

We would also like to thank Alison Barry, Dr Karin Lust, Dr Sophie Clarke, Barbara Waters, Kellie Himstedt, Dr Jon Hyett, and Dr Lynda Gillen for their support and contribution to the project and the midwives from Maternity Outpatient Department (MOPD), the birth suite and the labour ward. Funding

The BAMBINO project was supported by a Royal Brisbane and Women's Hospital foundation strategic initiative grant.

\section{Author details}

'Department of Internal Medicine and Aged Care, Royal Brisbane and Women's Hospital, (Butterfield St), Brisbane, (4029), Australia. ${ }^{2}$ Department of Epidemiology, Queensland Institute of Medical Research and School of 
Population Health, University of Queensland, (Herston Rd), Brisbane, (4029), Australia. ${ }^{3}$ School of Human Movement Studies and Institute of Health and Biomedical Innovation, Queensland University of Technology, Musk Ave, Brisbane,(4059), Australia. ${ }^{4}$ Department of Endocrinology and Obstetric Medicine, Mater Health Services and Mater Clinical School, University of Queensland, Stanley St, Brisbane, (4101), Australia. ${ }^{5}$ Department of Internal Medicine Services, Royal Brisbane and Women's Hospital and School of Medicine, University of Queensland, (Herston Rd) Brisbane, (4029), Australia.

\section{Authors' contributions}

KFF: Has been involved in design of the study, data collection, analysis and drafting the manuscripts. IJR has made substantial contributions to the statistical analysis of data and drafting of the manuscript. NMB has been involved in interpretation of the data and revising the intellectual content. HDM was involved in study design and revision of the intellectual content. LKC has been involved in the design of the study, obtained funding, provided oversight of the data collection and data analysis and revision of the intellectual content. All authors have read and approved the final manuscript.

\section{Authors' information}

KFF: MAppSc (Research), RN, RM.

IJR: BPsycSc (Hons), PhD. Postdoctoral Research Fellow at University of

Queensland and Queensland Institute of Medical Research.

NMB: BHMS, MAppSc, PhD.

HDM: MBBS (Hons), FRACP

LKC: MBBS (Hons), FRACP, PhD.

\section{Competing interests}

The authors declare that they have no competing interests.

Received: 8 April 2010 Accepted: 12 January 2011

Published: 12 January 2011

\section{References}

1. ACOG Committee opinion. Number 267, January 2002: exercise during pregnancy and the postpartum period. Obstetrics and gynecology 2002, 99(1):171-173.

2. Marquez-Sterling S, Perry AC, Kaplan TA, Halberstein RA, Signorile JF: Physical and psychological changes with vigorous exercise in sedentary primigravidae. Med Sci Sports Exerc 2000, 32(1):58-62.

3. Santos IA, Stein R, Fuchs SC, Duncan BB, Ribeiro JP, Kroeff LR, Carballo MT, Schmidt Ml: Aerobic exercise and submaximal functional capacity in overweight pregnant women: a randomized trial. Obstet Gynecol 2005, 106(2):243-249.

4. Clapp JF, Kim H, Burciu B, Schmidt S, Petry K, Lopez B: Continuing regular exercise during pregnancy: effect of exercise volume on fetoplacental growth. Am J Obstet Gynecol 2002, 186(1):142-147.

5. Hatch MC, Shu XO, McLean DE, Levin B, Begg M, Reuss L, Susser M: Maternal exercise during pregnancy, physical fitness, and fetal growth. American journal of epidemiology 1993, 137(10):1105-1114.

6. Ning Y, Williams MA, Dempsey JC, Sorensen TK, Frederick IO, Luthy DA: Correlates of recreational physical activity in early pregnancy. J Matern Fetal Neonatal Med 2003, 13(6):385-393.

7. Petersen $\mathrm{AM}$, Leet $\mathrm{TL}$, Brownson RC: Correlates of physical activity among pregnant women in the United States. Medicine and science in sports and exercise 2005, 37(10):1748-1753.

8. Wallace AM, Boyer DB, Dan A, Holm K: Aerobic exercise, maternal selfesteem, and physical discomforts during pregnancy. Journal of nursemidwifery 1986, 31(6):255-262.

9. Zhang J, Savitz DA: Exercise during pregnancy among US women. Annals of epidemiology 1996, 6(1):53-59.

10. Pereira MA, Rifas-Shiman SL, Kleinman KP, Rich-Edwards JW, Peterson KE, Gillman MW: Predictors of change in physical activity during and after pregnancy: Project Viva. American journal of preventive medicine 2007, 32(4):312-319.

11. Hinton PS, Olson CM: Predictors of pregnancy-associated change in physical activity in a rural white population. Maternal and child health journal 2001, 5(1):7-14.
12. Lacasse A, Rey E, Ferreira E, Morin C, Berard A: Epidemiology of nausea and vomiting of pregnancy: prevalence, severity, determinants, and the importance of race/ethnicity. BMC pregnancy and childbirth 2009, 9:26.

13. Garshasbi A, Faghih Zadeh S: The effect of exercise on the intensity of low back pain in pregnant women. International journal of gynaecology and obstetrics: the official organ of the International Federation of Gynaecology and Obstetrics 2005, 88(3):271-275.

14. Mogren IM: Previous physical activity decreases the risk of low back pain and pelvic pain during pregnancy. Scandinavian journal of public health 2005, 33(4):300-306.

15. Callaway LK, Prins JB, Chang AM, Mclntyre HD: The prevalence and impact of overweight and obesity in an Australian obstetric population. The Medical journal of Australia 2006, 184(2):56-59.

16. Gore SA, Brown DM, West DS: The role of postpartum weight retention in obesity among women: a review of the evidence. Ann Behav Med 2003, 26(2):149-159.

17. Callaway LK, Colditz PB, Byrne NM, Lingwood BE, Rowlands IJ, Foxcroft K, Mclntyre HD: Prevention of gestational diabetes: feasibility issues for an exercise intervention in obese pregnant women. Diabetes Care 33(7):1457-1459.

18. Children's Health Development Foundation SA and Deakin University: Australian Guide to Healthy Eating. Services CDoHaF CCDoHaF. Victoria; 1998, Edited by Services.

19. Institute of Medicine: Nutrition during pregnancy, weight gain and nutritional supplements. Report of the subcommittee on nutritional status and weight gain during pregnancy, subcommittee on dietary intake and nutrient supplements during pregnancy and lactation, food and nutrition board. Press NA. Washington DC; 1990.

20. Chasan-Taber L, Schmidt MD, Roberts DE, Hosmer D, Markenson G, Freedson PS: Development and validation of a Pregnancy Physical Activity Questionnaire. Medicine and science in sports and exercise 2004, 36(10):1750-1760.

21. Colley RC, Hills AP, O'Moore-Sullivan TM, Hickman IJ, Prins JB, Byrne NM: Variability in adherence to an unsupervised exercise prescription in obese women. International journal of obesity (2005) 2008, 32(5):837-844.

22. Roland M, Fairbank J: The Roland-Morris Disability Questionnaire and the Oswestry Disability Questionnaire. Spine 2000, 25(24):3115-3124.

23. Callaway LK, Colditz PB, Byrne NM, Lingwood BE, Rowlands IJ, Foxcroft KF, Mclntyre HD, group ftB: Prevention of gestational diabetes:Feasability issues for an exercise intervention in obese pregnant women. Diabetes Care 2010

24. Adolfsson A, Larsson PG, Wijma B, Bertero C: Guilt and emptiness: Women's experiences of miscarriage. Health Care for Women International 2004, 25:543-560.

25. Maker C, Ogden J: The miscarriage experience: More than just a trigger to psychological morbidity. Psychology and Health 2003, 18:403-415.

26. Maker C, Ogden J: The miscarriage experience: More than just a trigger to psychological morbidity. Psychology and health 2003, 18:403-415.

27. Horns PN, Ratcliffe LP, Leggett JC, Swanson MS: Pregnancy outcomes among active and sedentary primiparous women. J Obstet Gynecol Neonatal Nurs 1996, 25(1):49-54.

28. King T, Kavanagh AM, Jolley D, Turrell G, Crawford D: Weight and place: a multilevel cross-sectional survey of area-level social disadvantage and overweight/obesity in Australia. International journal of obesity (2005) 2006, 30(2):281-287.

\section{Pre-publication history}

The pre-publication history for this paper can be accessed here: http://www.biomedcentral.com/1471-2393/11/4/prepub

\section{doi:10.1186/1471-2393-11-4}

Cite this article as: Foxcroft ${ }^{1}$ et al:: Exercise in obese pregnant women: The role of social factors, lifestyle and pregnancy symptoms. BMC Pregnancy and Childbirth 2011 11:4. 